\title{
ACE and UCP2 gene polymorphisms and their association with baseline and exercise-related changes in the functional performance of older adults
}

Justin W L Keogh, Barry R Palmer, Denise Taylor, Andrew E Kilding

Maintaining high levels of physical function is an important aspect of successful ageing. While muscle mass and strength contribute to functional performance in older adults, little is known about the possible genetic basis for the heterogeneity of physical function in older adults and in how older adults respond to exercise. Two genes that have possible roles in determining levels of muscle mass, strength and function in young and older adults are angiotensin-converting enzyme (ACE) and mitochondrial uncoupling protein 2 (UCP2). This study examined whether polymorphisms in these two individual genes were associated with baseline functional performance levels and/or the training-related changes following exercise in previously untrained older adults. Five-eight Caucasian older adults (mean age 69.8 years) with no recent history of resistance training enrolled in a 12 week program of resistance, balance and cardiovascular exercises aimed at improving functional performance. Performance in 6 functional tasks was recorded at baseline and after 12 weeks. Genomic DNA was assayed for the ACE intron 16 insertion/deletion (I/D) and the UCP2 G-866A polymorphism. Baseline differences among genotype groups were tested using analysis of variance. Genotype differences in absolute and relative changes in physical function among the exercisers were tested using a general linear model, adjusting for age and gender. The genotype frequencies for each of the studied polymorphisms conformed to the Hardy-Weinberg equilibrium. The ACE I/D genotype was significantly associated with mean baseline measures of handgrip strength (II $30.9 \square 3.01 \mathrm{v}$. ID $31.7 \square 1.48$ v. DD $29.3 \square 2.18 \mathrm{~kg}, \mathrm{p}<0.001$ ), 8ft Up and Go time (II 6.45־0.48 v. ID/DD $4.41 \square 0.19 \mathrm{~s}, \mathrm{p}<0.001$ ) and 6 min walk distance (II 458\28.7 v. ID/DD 546\12.1m, $p=0.008)$. The UCP2 G-866A genotype was also associated with baseline $8 \mathrm{ft}$ Up and Go

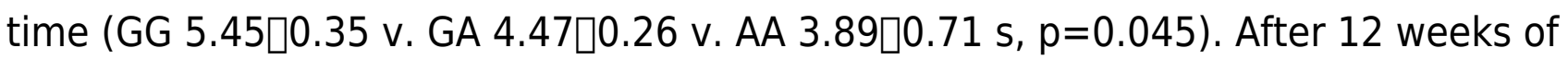
training, a significant difference between UCP2 G-886A genotype groups for change in $8 \mathrm{ft}$ Up and Go time was detected (GG $-0.68 \pm 0.17$ v. GA $-0.10 \pm 0.14$ v. AA +0.05 $\pm 0.31 \mathrm{~s}$, $p=0.023$ ). While several interesting and possibly consistent associations with older adults' baseline functional performance were found for the ACE and UCP2 polymorphisms, we found no strong evidence of genetic associations with exercise responses in this study. The 
relative equivalence of some of these training-response findings to the literature may have reflected the current study's focus on physical function rather than just strength, the relatively high levels of baseline function for some genotype groups as well as the greater statistical power for detecting baseline differences than the training-related changes. 
2 Justin W.L. Keogh ${ }^{1,2,3}$, Barry R. Palmer ${ }^{4,5}$, Denise Taylor ${ }^{6}$, Andrew E. Kilding ${ }^{2,7}$

3

$4 \quad{ }^{1}$ Faculty of Health Sciences and Medicine, Bond University, Australia

$5 \quad{ }^{2}$ Human Potential Centre, AUT University, Auckland, New Zealand

$6{ }^{3}$ Faculty of Science, Health, Education and Engineering, University of the Sunshine Coast, Australia

$7{ }^{4}$ Christchurch Heart Institute, Department of Medicine, University of Otago, Christchurch, New

8 Zealand

$9 \quad{ }^{5}$ College of Health, Massey University Wellington, New Zealand

$10 \quad{ }^{6}$ Health and Rehabilitation Research Institute, AUT University, Auckland, New Zealand

$11{ }^{7}$ School of Sport and Recreation, AUT University, Auckland, New Zealand

13 Running head: Genetic predictors of physical function

\section{Corresponding Author and Request for Reprints}

16 Justin Keogh

17 Faculty of Health Sciences and Medicine, Bond University, Australia

$18 \quad$ Tel: +61755954487

19 Fax: +61755954480

20 Email: jkeogh@,bond.edu.au 


\section{Abstract}

Maintaining high levels of physical function is an important aspect of successful ageing. While muscle mass and strength contribute to functional performance in older adults, little is known about the possible genetic basis for the heterogeneity of physical function in older adults and in how older adults respond to exercise. Two genes that have possible roles in determining levels of muscle mass, strength and function in young and older adults are angiotensin-converting enzyme $(A C E)$ and mitochondrial uncoupling protein $2(U C P 2)$. This study examined whether polymorphisms in these two individual genes were associated with baseline functional performance levels and/or the training-related changes following exercise in previously untrained older adults. Five-eight Caucasian older adults (mean age 69.8 years) with no recent history of resistance training enrolled in a 12 week program of resistance, balance and cardiovascular exercises aimed at improving functional performance. Performance in 6 functional tasks was recorded at baseline and after 12 weeks. Genomic DNA was assayed for the $A C E$ intron 16 insertion/deletion (I/D) and the $U C P 2$ G-866A polymorphism. Baseline differences among genotype groups were tested using analysis of variance. Genotype differences in absolute and relative changes in physical function among the exercisers were tested using a general linear model, adjusting for age and gender. The genotype frequencies for each of the studied polymorphisms conformed to the Hardy-Weinberg equilibrium. The $A C E$ I/D genotype was significantly associated with mean baseline measures of handgrip strength (II $30.9 \pm 3.01 \mathrm{v}$. ID $31.7 \pm 1.48 \mathrm{v}$. DD $29.3 \pm 2.18 \mathrm{~kg}, \mathrm{p}<0.001$ ), $8 \mathrm{ft}$ Up and Go time (II $6.45 \pm 0.48$ v. ID/DD $4.41 \pm 0.19 \mathrm{~s}, \mathrm{p}<0.001$ ) and 6 min walk distance (II $458 \pm 28.7 \mathrm{v}$. ID/DD 546 $\pm 12.1 \mathrm{~m}, \mathrm{p}=0.008)$. The $U C P 2 \mathrm{G}-866 \mathrm{~A}$ genotype was also associated with baseline $8 \mathrm{ft}$ Up and Go time (GG 5.45 \pm 0.35 v. GA $4.47 \pm 0.26$ v. AA $3.89 \pm 0.71 \mathrm{~s}, \mathrm{p}=0.045)$. After 12 weeks of training, a significant difference between UCP2 G-886A genotype groups for change in $8 \mathrm{ft}$ Up and Go time was detected (GG $-0.68 \pm 0.17$ v. GA $-0.10 \pm 0.14$ v. AA $+0.05 \pm 0.31 \mathrm{~s}, \mathrm{p}=0.023)$. While several interesting 
45 and possibly consistent associations with older adults' baseline functional performance were found for

46 the $A C E$ and $U C P 2$ polymorphisms, we found no strong evidence of genetic associations with exercise

47 responses in this study. The relative equivalence of some of these training-response findings to the

48 literature may have reflected the current study's focus on physical function rather than just strength, the

49 relatively high levels of baseline function for some genotype groups as well as the greater statistical

50 power for detecting baseline differences than the training-related changes. 
Introduction

52 Maintaining adequate levels of muscle mass, strength, muscular and aerobic endurance and functional

53 performance in older age is important, as a decline in these physical attributes may result in: 1) a loss

54 of independence (Baumgartner et al. 2004; Kim et al. 2012), 2) an increased risk and fear of falls

55 (Brouwer et al. 2004; Wagenaar et al. 2012); 3) an increase in the risk of chronic conditions (Abellan

56 van Kan et al. 2009); and 4) a reduction in quality of life (Giles et al. 2009; Masel et al. 2009).

57 Engaging in regular resistance and aerobic activity and ensuring adequate nutritional intake appear to

58 be some of the key strategies for older adults to reduce these sarcopenic-related losses of muscle mass,

59 strength and function (Fiatarone-Singh 2002; Nelson et al. 2007). While initial studies in this area

60 focused on maintaining muscle mass, a number of recent reviews indicate older adults at risk of

61 physical decline may gain more substantial improvements in muscular strength, balance and functional

62 performance, for example gait speed, than improve their muscle mass (Keogh \& MacLeod 2012;

63 Valenzuela 2012). This is vital as the age-related loss of strength, balance and gait speed has a greater

64 relationship to outcomes such as activities of daily living, independence and quality of life and

65 mortality than muscle mass alone (Abellan van Kan et al. 2009; Kim et al. 2012; Wood et al. 2011).

66 Studies involving younger (Argus et al. 2009; Till \& Cooke 2009) and middle-aged to older (Karavirta

67 et al. 2011) adults indicate the potential for considerable inter-individual responses to identical training

68 programs, even when the sample is relatively homogenous at baseline and engage in the same exercise

69 program. For example, in a study in which 175 untrained middle-aged to older adults performed 21

70 weeks of strength, aerobic training, combined strength and aerobic or no training, Karavirta et al.

71 (2011) reported large individual differences in muscular strength (-12 to 87\%) and aerobic power (-8 to

$7242 \%)$ changes for the combined strength and endurance training group $(n=53)$. This high degree of

73 within-participant variability in response was demonstrated via the use of criterion assessments of 
74 strength and aerobic power, namely the maximum voluntary contraction (MVC) and cycle ergometry 75 peak oxygen consumption $\left(\mathrm{VO}_{2 \text { peak }}\right)$ tests, respectively. Further, there were no significant correlations 76 (all r's $<0.10$ ) between the changes in the MVC strength or $\mathrm{VO}_{2 \text { peak }}$ in any of the three groups, 77 suggesting that the strength and aerobic power responses to strength and/or aerobic training can exhibit 78 substantial within- and between-participant variability. These intra-individual differences in training 79 response may reflect genetic factors.

80 While considerable heterogeneity exists in the physical function of many older adults, even when 81 chronic conditions and medications are controlled for, the relative contribution of genetics and long82 term physical activity levels in determining their physical function and ultimately survival remains less 83 clear. Based on research involving primarily younger adults, two genes that might play a role in 84 regulating the physical function and indirectly impact on survival in older adults are angiotensin85 converting enzyme $(A C E)$ and mitochondrial uncoupling protein 2 (UCP2) (Dhamrait et al. 2012;

86 Puthucheary et al. 2011).

87 When expressed, the $A C E$ gene produces angiotensin-converting enzyme protein. This $A C E$ protein 88 plays a key role in regulating the activity of the rennin-angiotensin system, thereby directly influencing 89 blood pressure and fluid balance and indirectly influencing cardiovascular and musculoskeletal 90 structure and function (Puthucheary et al. 2011; Seripa et al. 2011). Indeed, a functional polymorphism 91 in the human $A C E$ gene, the intron 16 insertion (I) allele, has been associated with an enhanced 92 cardiovascular response to training (Myerson et al. 1999), while the deletion (D) allele has been 93 associated with a superior muscle size and strength response to training (Puthucheary et al. 2011;

94 Woods et al. 2001). 
95 The UCP2 gene is expressed in many tissues particularly skeletal muscle. It exerts a variety of effects 96 on mitochondrial function, meaning it influences the rate of synthesis of ATP and reactive oxygen

97 species and indirectly influences many indices of cardiovascular health (Bo et al. 2013; Dato et al.

98 2014; Dhamrait et al. 2004; Palmer et al. 2003). The frequency of the UCP2 (A55V) C allele was

99 found to be higher among power athletes compared with controls (Sessa et al. 2011). Another allele of

100 the UCP2 G-866A polymorphism has also been associated with increased delta efficiency after

101 endurance training in young, healthy adults compared to those carrying the G allele (Dhamrait et al.

102 2012; Perusse et al. 2013).

103 Recently, some studies have examined the relationships between $A C E$ (Bustamante-Ara et al. 2010; 104 Garatachea et al. 2012; Giaccaglia et al. 2008; Pereira et al. 2013) and UCP2 (Dato et al. 2014) gene

105 polymorphisms and a variety of measures of muscular strength and power in older adults. Only one of

106 these four $A C E$ studies reported significant genotypic relationship to performance at baseline, with the

$107 A C E$ insertion/deletion (ACE ID) group having a significantly greater baseline handgrip strength

108 (Bustamante-Ara et al. 2010). In support of a possible genetic link to baseline function, Seripa et al.

109 (2011) demonstrated that the $A C E$ II genotype was associated with increased risk of limitations in

110 activities of daily living in hospitalised older patients. Of the two studies that assessed training-related

111 changes in older adults, both reported significantly greater improvements in strength and power

112 measures for those with the $A C E \mathrm{D}$ allele (Giaccaglia et al. 2008; Pereira et al. 2013). To the authors'

113 knowledge, only one study has so far reported associations between baseline differences in older adults

114 who differ in $U C P 2$ genotypes (Dato et al. 2014), with no studies examining the exercise response.

115 Dato et al. (2014) found significantly greater walking speeds over 3-4 $\mathrm{m}$ in those with the rs 7109266

116 SNP found on the UCP2 G-866A allele, but no UCP2 association with the degree of disability, 117 handgrip strength or cognitive function in a sample of 1089 older adults. 
118 Therefore, the purpose of this study was to determine if either the $A C E$ and/or $U C P 2$ genotypes

119 individually: 1) distinguish the baseline level of functional performance in older adults who are not

120 currently performing an exercise program; and 2) influence the training-related changes in older adults'

121 functional performance. It is hypothesised that variations in these individual alleles will be associated

122 with baseline- and training-related differences in older adults' functional performance.

123

124 Materials and Methods

\section{Research Design}

126 This study utilised a cross-sectional and pre-post single arm trial design to determine whether

127 polymorphisms in two individual genes ( $A C E$ and $U C P 2)$ were associated with baseline and training-

128 related changes in a variety of physical performance measures in community dwelling older adults,

129 respectively. Due to a lack of sufficient statistical power, no interactions between polymorphisms of

130 the two genes and the physical performance measures were examined. Portions of this data describing

131 the exercise program, physical assessments used and the magnitude of changes in physical function

132 seen with training have been reported previously (Keogh et al. 2014).

\section{Participants}

134 As the Never Too Old (N2O) program is a community-based program for older adults, there was no

135 specific inclusion/exclusion criteria for participation in this study, besides being at least 60 years of age

136 and healthy enough to be given medical clearance to participate in the program. Fifty-eight older adults

137 of European ancestry who had just enrolled in a $\mathrm{N} 2 \mathrm{O}$ program gave written informed consent to

138 participate in this study. These 58 participants were a subset of the 67 participants who gave informed 
139 consent to participate in Study 1 (which examined the training-related changes in physical function) of

140 the wider N2O study (Keogh et al. 2014). The nine participants whose data is not included in this

141 study either declined to participate in the current genetic project or were in eligible to participate based

142 on their ethnicity.

143 Prior to starting the N2O program, all subjects completed a modified PAR-Q pre-exercise health

144 assessment questionnaire to determine if the potential participants had any relative or absolute

145 contraindications to exercise. If the PAR-Q raised any concerns about the safety of exercise, the

146 individual was required to obtain clearance from a qualified medical practitioner prior to entry into the

147 program. Ethical clearance for the project was approved by the Auckland University of Technology

148 Human Ethics Committee (06/05).

\section{Training Program}

Participants attended the $\mathrm{N} 2 \mathrm{O}$ program twice weekly for 12 weeks, with each training session

151 lasting approximately 60 minutes. The participants in this research project performed the Bronze N20

152 program, which focused on providing a safe and friendly environment that introduced the older adults

153 to selected resistance and cardiovascular exercises that were aimed at improving their overall

154 functional performance and health.

155

156

157

158

159

160
Training sessions commenced with a 5-10 minute warm-up that preceded the resistance training component. The resistance training exercises included the knee extension, leg curl, leg press, chest press, lat pulldown, shoulder press, bicep curl and tricep pushdown, with a selection of these exercises performed during each training session. Each exercise was performed for 1-2 sets of 8-12 repetitions with loads that initially produced moderately light to moderate ratings of perceived on the Borg rating of perceived exertion (RPE) scale (Borg 1982). After completing the resistance training exercises, 5- 
16110 minutes of cardiovascular exercise (i.e. stationary cycling or walking on a treadmill) was then

162 performed at a moderately light to moderate intensity on the Borg RPE scale (Borg 1982). The

163 exercise session was then completed by performing 5-10 minutes of stretches for the major muscle

164 groups of the body. Loads for the resistance and cardiovascular exercises were progressively increased

165 over the course of the 12 week training program.

\section{Procedures}

168 The $\mathrm{N} 2 \mathrm{O}$ program is aligned with the International Society of Aging and Physical Activity (ISAPA) 169 and adopts the ISAPA's recommended Senior Fitness Test battery (Rikli \& Jones 1999a; Rikli \& Jones 170 1999b; Rikli \& Jones 2001). This series of assessments has been to shown to be reliable and valid in 171 predicting functional levels in older adults and involves the: 1) $30 \mathrm{~s}$ sit to stand (30 s STS); 2) $30 \mathrm{~s}$

172 bicep curl; 3) $8 \mathrm{ft} \mathrm{Up}$ and Go; and 4) 6 min walk tests. The 30 s STS and bicep curl stand tests were 173 designed to assess lower and upper limb muscular strength and endurance, respectively of older adults 174 (Rikli \& Jones 1999a; Rikli \& Jones 1999b). The $8 \mathrm{ft} \mathrm{Up}$ and Go and the 6 min walk tests assess 175 dynamic balance/mobility and walking endurance, respectively (Rikli \& Jones 1999a; Rikli \& Jones 176 1999b). Measures of upper limb dexterity (Purdue Pegboard Test) and strength (handgrip strength)

177 were also assessed, as older adults have reduced upper limb dexterity and strength compared to young 178 adults (Keogh et al. 2007; Sequeria et al. 2012). In addition, the height and body mass of each

179 participant was also obtained using standard procedures so that a body mass index (BMI) could be 180 calculated. BMI was calculated by dividing the body mass in $\mathrm{kg}$ by the square of the height in metres.

181 All of these tests were conducted within the same assessment session that were completed in $<1$ hour in 182 the same order as described above with the exception that the 6 min walk test was performed last due 
183 to its fatiguing nature. Prior to performing these eight assessments, all participants completed a 5-10

184 min warm-up that consisted of general total body movements and stretches of primary muscle groups.

185 A description of the eight tests is provided below. Prior to performing each of the tests, participants

186 were given the same instructions, these being "to do the best they can on the tests, but to never push

187 themselves to the point of overexertion or beyond what they think is safe for them" (Rikli \& Jones

188 1999a).

18930 s STS: The participant was asked to fully stand and sit down as many times as possible in $30 \mathrm{~s}$ from

190 a chair without arms, a seat height of $43 \mathrm{~cm}$ and with their arms crossed at the wrist and held against

191 the chest. One practice trial, of 2-3 repetitions, was performed as a specific warm-up followed by one

192 full trial of this test. The score was the complete number of correct repetitions performed in $30 \mathrm{~s}$.

$19330 \mathrm{~s}$ Bicep Curl: The participant was asked to sit down on a chair and to complete as many bicep curls

194 in $30 \mathrm{~s}$ as possible with their preferred arm. This test was done with $2 \mathrm{~kg}$ or $4 \mathrm{~kg}$ dumbbells for older

195 women and men, respectively. The participant was required to keep their trunk still and to move their

196 forearm through a full range of motion about the elbow joint for each repetition to be counted. One

197 practice trial of 2-3 repetitions (if possible) were performed as a specific warm-up followed by one full

198 trial of this test. The score was the complete number of correct repetitions performed in $30 \mathrm{~s}$.

$199 \underline{8 \mathrm{ft} \mathrm{Up} \text { and Go: }}$ The $8 \mathrm{ft} \mathrm{Up}$ and Go required the participant who was initially seated on a chair that 200 was $\sim 43 \mathrm{~cm}$ high and no arm rests, to stand up, walk around a cone positioned 8 feet $(\sim 2.44 \mathrm{~m})$ away 201 and sit down again in the chair. One practice trial was performed as a specific warm-up followed by 202 two trial of this test, each separated by a rest period of $60 \mathrm{~s}$. The time required to complete each trial of 203 this test was measured via stopwatch to the nearest $0.01 \mathrm{~s}$. 
204 6 Min Walk: The 6 min walk test was performed by having several older adults starting at different 205 points of a $50 \mathrm{~m}$ rectangular course. This course was marked with cones every $5 \mathrm{~m}$ to facilitate easy 206 recording of distance walked. One trial was performed due to the challenging and fatiguing nature of 207 this test.

208 Purdue Pegboard Test: (PPT) The upper limb dexterity of the older adults was assessed by the PPT 209 (Tiffin \& Asher 1948). The PPT is a time-based dexterity assessment tool comprising four sub-tasks; 210 right hand only, left hand only, both hands and an assembly task, in which the participant is required to 211 place the maximum number of pegs in the holes on the pegboard in a set time. For the handed tests, the 212 participant was required to place pegs into evenly spaced holes on the board using the indicated hand.

213 For the assembly task there is an additional component with the assembly made up of the pegs, 214 washers and collars, placed in a specific order. The individual and both handed tasks are performed for $21530 \mathrm{~s}$ while the assembly task allows $60 \mathrm{~s}$ due to its greater complexity. Each of the sub-tasks was 216 performed 2 times to reduce possible learning effect and improve the reliability of the test (Tiffin \& 217 Asher 1948).

218 Isometric Handgrip Strength: Isometric MVC grip strength of the preferred limb was assessed with a 219 hand-grip dynamometer (Bohannon 2002). Participants performed one practice and two test trials of 220 the isometric hand-grip strength test. For all trials, the preferred arm had to be kept by the side of the 221 participant with the elbow maintained at $\sim 90^{\circ}$ of elbow flexion. A rest period of $60 \mathrm{~s}$ was given 222 between each trial.

\section{DNA extraction and genotyping}

224 For genetic analysis buccal cells were harvested from participants via a mouthwash of $10 \mathrm{ml} 4 \%$ 225 sucrose in sterile deionised water. The cell suspension was recovered in a $50 \mathrm{ml}$ tube, vortexed 
226 vigorously and stored at $-20^{\circ} \mathrm{C}$ until DNA isolation was performed as previously reported (Palmer \& 227 Healy 1993).

228 DNA samples (100 ng) were genotyped for the $A C E$ intron 16 insertion/deletion (I/D) polymorphism 229 using a two primer polymerase chain reaction (PCR) protocol (Rigat et al. 1992) with the addition of $2305 \%$ DMSO to eliminate mis-amplification of heterozygous templates (Fogarty et al. 1994). A PCR231 RFLP assay (Esterbauer et al. 2001) was employed to genotype $100 \mathrm{ng}$ DNA samples for the UCP2 G$232866 \mathrm{~A}$ polymorphism, in which $360 \mathrm{bp}$ amplimers were digested with MluI. Amplimers were 233 electrophoresed on 1 or $2 \%$ agarose, $0.5 \times$ TBE gels stained with ethidium bromide and visualized with 234 a Bio-Rad Fluor-S imaging system (Hercules, California). A random subset (20\%) of the samples were 235 regenotyped in separate assays with $100 \%$ concordance.

\section{Statistical Analysis}

237 Data are presented as mean \pm standard deviation (SD). Data were analysed using a statistical software 238 package (SPSS, v14, Chicago). Baseline differences among genotype groups were tested using analysis 239 of variance. Genotype differences in absolute and relative changes in physical function among the 240 exercisers were tested using a general linear model, adjusting for age and gender. Additive and D 241 allele-dominant $(A C E)$ and additive and A allele-dominant (UCP2) genetic models were all evaluated.

242 Statistical significance was set at $p<0.05$. As calculated using G*Power 3.1.3 (Faul et al. 2009), the 243 study had greater than $90 \%$ power to detect a difference in (for example) baseline Up \& Go 244 performance between the $A C E \mathrm{DD} / \mathrm{ID}$ and II genotype groups with an $\alpha=0.05$. However the study 245 only had $70 \%$ power to detect a difference in post-training $8 \mathrm{ft} \mathrm{Up} \mathrm{\&} \mathrm{Go} \mathrm{performance} \mathrm{between} \mathrm{these}$ 246 groups with an $\alpha=0.05$. 


\section{Results}

249 A description of the participants' baseline demographic data is provided in Table 1. Almost all 250 participants had at least one chronic disease, with high blood pressure (19\%), arthritis and other joint 251 conditions $(16 \%)$ and cardiovascular disease $(12 \%)$ being most common. At baseline, most 252 participants were somewhat physically active (i.e. performed $\sim 60$ minutes of moderate physical activity

253 per week) and possessed moderately low to moderately high levels of physical function, based on age254 and gender-matched normative data for the Senior Fitness Test (Rikli \& Jones 1999b). Program 255 attendance was very high with the participants attending $22 \pm 2$ out of the requested 24 training 256 sessions, resulting in an overall attendance rate of $93 \%$.

257 Insert Table 1 about here

258 DNA samples were successfully obtained from all participants who commenced the study. However, a 259 small number of subjects did not complete all physical tests during the pre- and post-training 260 assessments. Therefore the actual sample size for each test differed somewhat for each of the tests, 261 with this presented within the Tables. Importantly, the resulting genotype frequencies for each of the 262 studied polymorphisms conformed to the Hardy-Weinberg equilibrium (Table 2).

263 Insert Table 2 about here

\section{Characteristics and baseline phenotypes}

265 Overall, the frequencies of the $A C E$ genotypes were 21,49 and $30 \%$ for II, ID and DD. The 266 distribution of $U C P 2$ G-8661 genotypes was 34, 54 and $12 \%$ for GG, GA and AA respectively. There 267 were no differences in age, gender, or baseline BMI between genotype groups. As such, all data 268 reported in the tables is for the overall group of 58 male and female participants. However, significant 
269 differences were observed for some baseline measures of physical function, when adjusted for age and 270 gender (Tables 3 and 4), specifically between some $A C E$ genotype groups for the 6 min walk, $8 \mathrm{ft} \mathrm{Up} \&$

271 Go and handgrip strength (Table 3) and $U C P 2$ genotype groups for $8 \mathrm{ft} \mathrm{Up} \&$ Go and handgrip strength 272 (Table 4).

273 Insert Tables 3 and 4 about here

\section{Intra- and inter-genotype response to physical training response}

275 Forty-three percent of the overall sample completed all 24 training sessions. Mean attendance of the 276 programme was $22 \pm 2$ out of 24 sessions (93\%). No significant difference in attendance rates were 277 observed between male and female participants (data not shown).

278 Tables 5 and 6 show the absolute changes for each physical measure after 12 weeks of training for each 279 genotype group. A trend towards improvement in muscle strength (bicep curls) was observed between $280 A C E$ II v. ID/DD ( $\mathrm{p}=0.099)$. There was a significant difference between UCP2 G-886A genotype 281 groups for the $8 \mathrm{ft} \mathrm{Up}$ and Go test $(\mathrm{p}=0.023)$. Bonferroni correction for multiple testing suggested these 282 differences may be due to chance, as $\mathrm{P}>0.05$ after correction.

283 Insert Tables 5 and 6 about here

\section{Discussion}

286 The current study's primary aims were to determine if baseline differences and training-related changes 287 in functional performance were related to particular genotypes of the $A C E$ and $U C P 2$ genes in older 288 adults. The frequencies of the $A C E$ and $U C P 2$ G-8661 genotypes in our sample conformed to the 
289 expectations of the Hardy-Weinberg equilibrium and to that of a New Zealand population of Caucasian 290 ancestry (Palmer et al. 2009; Palmer et al. 2003).

291 At baseline, it was observed that the $A C E$ gene variant genotypes were associated with significant 292 differences in 3 of the 6 performance tests (6 min walk, 8ft Up and Go and handgrip strength). 293 Baseline variations in the UCP2 gene polymorphism were also observed for the $8 \mathrm{ft}$ Up and Go, with 294 handgrip strength approaching significance $(\mathrm{p}=0.094)$.

295 The ID/DD $A C E$ allele groups performed significantly better at baseline than those with those with II 296 alleles on the 6 min walk and 8ft Up and Go tests. While these two walking tests both require the 297 participants to walk as quickly as possible over a set course, these tests differ in several ways. The 6 298 min walk test requires the participants to walk a maximum distance in 6 minutes, and is therefore 299 considered a field test of $\mathrm{VO}_{2 \text { peak }}$ and walking ability for older adults (Rikli \& Jones 1999a; Rikli \& 300 Jones 1999b). In contrast, the $8 \mathrm{ft} \mathrm{Up}$ and Go requires the participants to stand up, walk a distance of 8 301 feet, turnaround, walk back to the chair and then sit down, suggesting a greater reliance on lower body 302 strength, walking speed and mobility (Rikli \& Jones 1999a; Rikli \& Jones 1999b).

303 The baseline differences for the 8ft Up and Go appear consistent with previous studies demonstrating 304 increased muscular strength or power with the $\mathrm{D}$ allele of the $A C E$ gene for younger adults 305 (Puthucheary et al. 2011; Woods et al. 2001), but in contrast to some studies involving older adults 306 (Garatachea et al. 2012; Giaccaglia et al. 2008; Pereira et al. 2013). While the greater 6 minute walk 307 distance for the ID/DD groups was initially unexpected, a lack of lower body strength may reduce the 308 ability of older adults to walk briskly for extended periods of time. Research support for this view is 309 provided by the significant improvements in $\mathrm{VO}_{2 \text { peak }}$ observed in older adults as a consequence of 310 resistance training (Lovell et al. 2009). The significantly greater performance in the two walking tests 
311 for the ID/DD groups is also consistent with a recent report (Seripa et al. 2011), observing that the $A C E$

312 II genotype was associated with greater limitations in activities of daily living in hospitalised older

313 patients. As gait speed over a variety of distances is an influential determinant of maintaining 314 independence and in reducing many age-related adverse effects (Abellan van Kan et al. 2009), the 315 results of our study and Serpia et al. (2011) suggest some genetic component to the wide variation seen 316 in walking speed and performance of older adults (Peel et al. 2013).

317 Based on the reported role of the $A C E$ gene in human physical performance studies involving younger 318 adults (Dhamrait et al. 2012; Puthucheary et al. 2011), our findings at baseline for the 30 s STS and 319 handgrip strength test were somewhat unexpected, with greater performance expected from those with 320 the ID/DD alleles for both of these tests. A significant between-group was observed for handgrip 321 strength, whereby the ID group had greater handgrip strength than the DD group. While such a result 322 was not expected based on the results of the younger adult literature, it was consistent with the only

323 other study conducted to date involving older adults (Bustamante-Ara et al. 2010). Our results 324 indicated no significant baseline differences between $A C E$ genotype groups for the $30 \mathrm{~s}$ STS. While 325 this may have unexpected based on the younger adult literature, the lack of significant baseline 326 differences in $30 \mathrm{~s}$ STS performance was also consistent with the results reported for 139 older women 327 by Pereira et al. (2013).

328 Comparisons of the baseline performance results for the UCP2 genotype groups also revealed 329 significant differences for the $8 \mathrm{ft} \mathrm{Up}$ and Go test and a trend for handgrip strength $(\mathrm{p}=0.094)$, 330 whereby greater performance was found in those with the A than G UCP2 allele. This result was 331 somewhat consistent with (Dato et al. 2014), who reported significantly greater walking speeds over 3$3324 \mathrm{~m}$ in older adults with the UCP2 A allele. However, the direction of these baseline differences for 333 the $8 \mathrm{ft} \mathrm{Up}$ and Go test was inconsistent with the greater improvements in endurance performance 
334 markers for the A compared to $\mathrm{G}$ allele carriers in younger populations (Dhamrait et al. 2012; Perusse 335 et al. 2013). Such inconsistencies in the association between UCP2 polymorphisms and endurance vs 336 power performance in younger compared to older adults warrants further investigation.

337 The second aim of the current study was to examine whether the $A C E$ and $U C P 2$ gene influenced the 338 training related responses in older adults' functional performance. The training program used in the 339 current study appeared effective as the magnitude of the training related changes for the Functional 340 Fitness Test battery outcomes was relatively consistent with that reported for other community-based 341 older adult exercise programs (Bates et al. 2009; Belza et al. 2010; Henwood et al. 2013). Inspection 342 of the data revealed little evidence for the $A C E$ or $U C P 2$ gene alleles to have any significant effects on 343 the training-related changes in the outcome measures assessed in this study. Exceptions to this rule 344 were the significantly greater improvements for the GG UCP2 group in the $8 \mathrm{ft}$ Up and Go and the 345 trend $(\mathrm{p}=0.099)$ for larger improvements in bicep curl strength for the ID/DD versus II $A C E$ allele 346 groups. These associations between the $U C P 2$ gene and training-related changes in the $8 \mathrm{ft} \mathrm{Up}$ and Go 347 test are novel and to the authors' knowledge, our study is the first to assess the relationship between the $348 U C P 2$ genotypes and changes in physical performance in older adults.

349 The lack of differences in the training related response between different $A C E$ genotype groups was 350 consistent with the only study to assess changes in older adults' 6 minute walk performance resulting 351 from 18 months of combined aerobic and resistance training (Giaccaglia et al. 2008). The responses in 352 our assessments that were more dependent on strength or power were inconsistent with the two studies 353 conducted to date, where significantly greater improvements in muscular strength and power were 354 associated with the $A C E \mathrm{D}$ gene allele (Giaccaglia et al. 2008; Pereira et al. 2013). Potential reasons 355 for the variation in our results compared to the two studies within the literature may be explained by 356 three primary factors. 
357 The first of these was the difference in outcome measures assessed in the current study compared to 358 that of the literature. The current study focused on functional performance tests ( $30 \mathrm{~s}$ STS, bicep curl, $3598 \mathrm{ft} \mathrm{Up}$ and Go and 6 minute walk), whereas most of the outcomes assessed in the literature were 360 strength or power tests. While performance in many of the functional performance tests we selected is 361 moderately correlated with strength and/or $\mathrm{VO}_{2 \text { peak }}$, our assessments were not criterion measures of 362 either of these physical qualities. It is therefore likely that functional performance tests have a greater 363 variety of determinants than criterion tests of muscular strength or $\mathrm{VO}_{2 \text { peak }}$, thereby reducing the 364 potential for isolated genotypes to significantly influence training-related changes in functional 365 performance. Such speculation is consistent with the lack of any significant relationship between the $366 A C E$ and $U C P 2$ genotypes for the older adults' baseline- or training-related differences in upper limb 367 dexterity (Purdue Pegboard) test performance seen in this study. The second difference may have been 368 the relatively high levels of baseline function for some genotype groups in the current study compared 369 to that of the literature. Specifically, the $A C E$ ID/DD and $U C P 2$ AA gene allele groups who had the 370 highest scores at baseline, were typically in the $60-80^{\text {th }}$ percentile (men) and $80-90^{\text {th }}$ percentiles 371 (women) for the $30 \mathrm{~s}$ STS, bicep curl, 6 min walk and $8 \mathrm{ft} \mathrm{Up}$ and Go tests (Rikli \& Jones 1999b). In 372 comparison, our other gene allele group and older adults in the other studies within this literature appeared to have much poorer baseline function. These weaker older adults in ours and other studies therefore had more chance to demonstrate significant improvements in function and significant genotype-related responses to training than our higher functioning ID/DD and UCP2 AA groups. Thirdly, the current study had more modest statistical power for detecting training-related changes ( $70 \%$ power) than baseline differences ( $90 \%$ power).

In conclusion, the current study adds to the literature on the potential link between genotype and physical performance in older adults. While our baseline results were generally consistent with the phenotypes associated with the $A C E$ I/D and $U C P 2$ polymorphisms, we found no strong evidence of 
381 genetic association to the functional performance response to physical training in this study. Some of 382 this relative equivalence may have reflected our focus on functional performance such as the $8 \mathrm{ft} \mathrm{Up}$ 383 and Go test rather than physical capacity outcomes e.g. MVC strength or aerobic power. Specifically, 384 functional performance assessments would appear to require the integration of a greater number of 385 sensoriomotor functions than physical capacity assessments of $\mathrm{MVC}$ or $\mathrm{VO}_{2 \text { peak }}$. The variance in 386 results for this study compared to that of literature may also reflect the relatively high levels of baseline 387 function for some genotype groups of the older adults in the current study as well as the greater 388 statistical power for detecting baseline differences ( $90 \%$ power) than the training-related changes ( $70 \%$ 389 power). Future studies in this area should recruit larger sample sizes so to obtain sufficient statistical 390 power to detect meaningful training related responses in a variety of health, physical fitness and 391 functional performance outcomes and ensure no statistically significant between-group differences in 392 outcome scores at baseline. These future studies should also be sufficiently powered to detect 393 differences in training response as a function of interactions between different polymorphisms of 394 multiple genes that may play a role in modulating the exercise response. 
Abellan van Kan G, Rolland Y, Andrieu S, Bauer J, Beauchet O, Bonnefoy M, Cesari M, Donini LM, Gillette Guyonnet S, Inzitari M, Nourhashemi F, Onder G, Ritz P, Salva A, Visser M, and Vellas B. 2009. Gait speed at usual pace as a predictor of adverse outcomes in communitydwelling older people an International Academy on Nutrition and Aging (IANA) Task Force.

Argus CK, Gill ND, Keogh JWL, Hopkins WG, and Beaven CM. 2009. Changes in strength, power 403 and steroid hormones during a professional rugby union competition. Journal of Strength and Conditioning Research 23:1583-1592.

405

406

407

408

409

410

411

412

413

414

415

416

417 Journal of Nutrition, Health and Aging 13:881-889.

\section{Conditioning Research 23:1583-1592.}

Bates A, Donaldson A, Lloyd B, Castell S, Krolik P, and Coleman R. 2009. Staying active, staying strong: pilot evaluation of a once-weekly, community-based strength training program for older adults. Health Promotion Journal of Australia 20:42-47.

Baumgartner RN, Wayne SJ, Waters DL, Janssen I, Gallagher D, and Morley JE. 2004. Sarcopenic obesity predicts instrumental activities of daily living disability in the elderly. Obesity Research 12:1995-2004.

Belza B, Snyder S, Thompson M, and LoGerfo J. 2010. From Research to Practice: EnhanceFitness, an Innovative Community-Based Senior Exercise Program. Topics in Geriatric Rehabilitation 26:299-309 210.1097/TGR.1090b1013e3181fee1069e.

Bo H, Jiang N, Ji LL, and Zhang Y. 2013. Mitochondrial redox metabolism in aging: Effect of exercise interventions. Journal of Sport and Health Science 2:67-74.

Bohannon RW. 2002. Quantitative testing of muscle strength: issues and practical options for the geriatric population. Topics in Geriatric Rehabilitation 18:1-17. 
418 Borg GA. 1982. Psychophysical bases of perceived exertion. Medicine and Science in Sports and Exercise 14:377-381.

420 Brouwer B, Musselman K, and Culham E. 2004. Physical function and health status among seniors with and without a fear of falling. Gerontology 50:135-141.

422 Gil P, Serra-Rexach JA, Ruiz JR, and Lucia A. 2010. ACE and ACTN3 genes and muscle phenotypes in nonagenarians. International Journal of Sports Medicine 31:221-224.

Dato S, Soerensen M, Lagani V, Montesanto A, Passarino G, Christensen K, Tan Q, and Christiansen L. 2014. Contribution of genetic polymorphisms on functional status at very old age: a genebased analysis of 38 genes (311 SNPs) in the oxidative stress pathway. Exp Gerontol 52:23-29.

Dhamrait SS, Stephens JW, Cooper JA, Acharya J, Mani AR, Moore K, Miller GJ, Humphries SE, Hurel SJ, and Montgomery HE. 2004. Cardiovascular risk in healthy men and markers of oxidative stress in diabetic men are associated with common variation in the gene for uncoupling protein 2. European Heart Journal 25:468-475.

Dhamrait SS, Williams AG, Day SH, Skipworth J, Payne JR, World M, Humphries SE, and Montgomery HE. 2012. Variation in the uncoupling protein 2 and 3 genes and human performance. Journal of Applied Physiology 112:1122-1127.

Esterbauer H, Schneitler C, Oberkofler H, Ebenbichler C, Paulweber B, Sandhofer F, Ladurner G, Hell E, Strosberg AD, Patsch JR, Krempler F, and Patsch W. 2001. A common polymorphism in the promoter of UCP2 is associated with decreased risk of obesity in middle-aged humans. Nature Genetics 28:178-183.

Faul F, Erdfelder E, Buchner A, and Lang A-G. 2009. Statistical power analyses using G*Power 3.1: Tests for correlation and regression analyses. Behavior Research Methods 41:1149-1160. 
441 Fiatarone-Singh MA. 2002. Exercise comes of age: rationale and recommendations for a geriatric

442 exercise prescription. Journals of Gerontology Series A, Biological Sciences and Medical Sciences 57A:M262-M282.

Fogarty DG, Maxwell AP, Doherty CC, Hughes AE, and Nevin NC. 1994. ACE gene typing. Lancet 343:851.

Garatachea N, Fiuza-Luces C, Torres-Luque G, Yvert T, Santiago C, Gomez-Gallego F, Ruiz JR, and Lucia A. 2012. Single and combined influence of ACE and ACTN3 genotypes on muscle phenotypes in octogenarians. European Journal of Applied Physiology 112:2409-2420.

Giaccaglia V, Nicklas B, Kritchevsky S, Mychalecky J, Messier S, Bleecker E, and Pahor M. 2008. Interaction between angiotensin converting enzyme insertion/deletion genotype and exercise training on knee extensor strength in older individuals. International Journal of Sports Medicine 29:40-44.

Giles L, Hawthorne G, and Crotty M. 2009. Health-related Quality of Life among hospitalized older people awaiting residential aged care. Health and Quality of Life Outcomes 7:71.

Henwood T, Wooding A, and de Souza D. 2013. Centre-based exercise delivery: Feasibility of a staff delivered program and the benefits for low-functioning older adults accessing respite day care. Activities, Adaptation \& Aging 37:224-238.

Karavirta L, Hakkinen K, Kauhanen A, Arija-Blazquez A, Sillanpaa E, Rinkinen N, and Hakkinen A. 2011. Individual responses to combined endurance and strength training in older adults. Medicine and Science in Sports and Exercise 43:484-490.

Keogh JW, Morrison S, and Barrett R. 2007. Strength-training improves the tri-digit finger-pinch force control of older adults. Archives of Physical Medicine and Rehabilitation 88:1055-1063. 
463 Keogh JW, Rice J, Taylor D, and Kilding A. 2014. Objective and subjective benefits of a community-

464

465

466

467

468

469

470

471

472

473

474

475

476

477

478

479

480

481

482

483

484

485

486 based, older adult multi-component exercise programme. Journal of Primary Health Care 6:114-122.

Keogh JWL, and MacLeod RD. 2012. Body composition, physical fitness, functional performance, quality of life and fatigue benefits of exercise for prostate cancer patients: a systematic review. Journal of Pain and Symptom Management 43:96-110.

Kim KE, Jang SN, Lim S, Park YJ, Paik NJ, Kim KW, Jang HC, and Lim JY. 2012. Relationship between muscle mass and physical performance: is it the same in older adults with weak muscle strength? Age and Ageing 41:799-803.

Lovell DI, Cuneo R, and Gass GC. 2009. Strength training improves submaximum cardiovascular performance in older men. Journal of Geriatric Physical Therapy 32:117-124.

Masel M, Graham J, Reistetter T, Markides K, and Ottenbacher K. 2009. Frailty and health related quality of life in older Mexican Americans. Health and Quality of Life Outcomes 7:70.

Myerson S, Hemingway H, Budget R, Martin J, Humphries S, and Montgomery H. 1999. Human angiotensin I-converting enzyme gene and endurance performance. Journal of Applied Physiology 87:1313-1316.

Nelson ME, Rejeski WJ, Blair SN, Duncan PW, Judge JO, King AC, Macera CA, and CastanedaSceppa C. 2007. Physical activity and public health in older adults: recommendation from the American College of Sports Medicine and the American Heart Association. Medicine and Science in Sports and Exercise 39:1435-1445.

Palmer B, and Healy J. 1993. Development of a practical illustrating the use of the polymerase chain reaction for genetic testing. Biochemical Education 21:106-107.

Palmer BR, Devereaux CL, Dhamrait SS, Mocatta TJ, Pilbrow AP, Frampton CM, Skelton L, Yandle TG, Winterbourn CC, Richards AM, Montgomery HE, and Cameron VA. 2009. The common 
G-866A polymorphism of the UCP2 gene and survival in diabetic patients following myocardial infarction. Cardiovascular Diabetology 8:31.

Palmer BR, Pilbrow AP, Yandle TG, Frampton CM, Richards AM, Nicholls MG, and Cameron VA. 2003. Angiotensin-converting enzyme gene polymorphism interacts with left ventricular ejection fraction and brain natriuretic peptide levels to predict mortality after myocardial infarction. Journal of the American College of Cardiology 41:729-736.

Peel NM, Kuys SS, and Klein K. 2013. Gait speed as a measure in geriatric assessment in clinical settings: a systematic review. Journals of Gerontology Series A, Biological Sciences and Medical Sciences 68:39-46.

Pereira A, Costa AM, Izquierdo M, Silva AJ, Bastos E, and Marques MC. 2013. ACE I/D and ACTN3 $\mathrm{R} / \mathrm{X}$ polymorphisms as potential factors in modulating exercise-related phenotypes in older women in response to a muscle power training stimuli. Age 35:1949-1959.

Perusse L, Rankinen T, Hagberg JM, Loos RJ, Roth SM, Sarzynski MA, Wolfarth B, and Bouchard C. 2013. Advances in exercise, fitness, and performance genomics in 2012. Medicine and Science in Sports and Exercise 45:824-831.

Puthucheary Z, Skipworth JA, Rawal J, Loosemore M, Van Someren K, and Montgomery H. 2011. The ACE Gene and Human Performance. Sports Medicine 41:433-448.

Rigat B, Hubert C, Corvol P, and Soubrier F. 1992. PCR detection of the insertion/deletion polymorphism of the human angiotensin converting enzyme gene (DCP1) (dipeptidyl carboxypeptidase 1). Nucleic Acids Research 20:1433-1433.

Rikli RE, and Jones CJ. 1999a. Development and validation of a functional fitness test for communityresiding older adults. Journal of Aging and Physical Activity 7:129-161.

Rikli RE, and Jones CJ. 1999b. Functional fitness normative scores for community-residing older adults, ages 60-94. Journal of Aging and Physical Activity 7:162-181. 
511 Rikli RE, and Jones CJ. 2001. Senior Fitness Test Manual. Champaign, IL: Human Kinetics.

512 Sequeria G, Keogh JW, and Kavanagh JJ. 2012. Resistance training can improve fine manual dexterity 513 in essential tremor patients: a preliminary study. Archives of Physical Medicine and 514 Rehabilitation 93:1466-1468.

515 Seripa D, Paroni G, Matera MG, Gravina C, Scarcelli C, Corritore M, D'Ambrosio LP, Urbano M, 516 D'Onofrio G, Copetti M, Kehoe PG, Panza F, and Pilotto A. 2011. Angiotensin-converting enzyme (ACE) genotypes and disability in hospitalized older patients. Age 33:409-419.

Sessa F, Chetta M, Petito A, Franzetti M, Bafunno V, Pisanelli D, Sarno M, Iuso S, and Margaglione M. 2011. Gene polymorphisms and sport attitude in Italian athletes. Genetic Testing and Molecular Biomarkers 15:285-290.

Tiffin J, and Asher EF. 1948. The Purdue Pegboard: norms and studies of reliability and validity. Journal of Applied Psychology 32:234-247.

Till KA, and Cooke C. 2009. The effects of postactivation potentiation on sprint and jump performance of male academy soccer players. Journal of Strength and Conditioning Research 23:1960-1967.

Valenzuela T. 2012. Efficacy of progressive resistance training interventions in older adults in nursing homes: a systematic review. Journal of the American Medical Directors Association 13:418428.

Wagenaar R, Keogh JWL, and Taylor D. 2012. Development of a clinical Multiple-Lunge test to predict falls in older adults. Archives of Physical Medicine and Rehabilitation 93:458-465.

Wood JL, Iuliano-Burns S, King SJ, Strauss BJ, and Walker KZ. 2011. Poor physical function in elderly women in low-level aged care is related to muscle strength rather than to measures of sarcopenia. Clinical Interventions in Aging 6:67-76. 
533 Woods D, Hickman M, Jamshidi Y, Brull D, Vassiliou V, Jones A, Humphries S, and Montgomery H. 2001. Elite swimmers and the D allele of the ACE I/D polymorphism. Human Genetics 108:230-232.

536 


\section{Table 1 (on next page)}

Baseline demographic data for the sample 
2 Table 1. Baseline demographic data for the sample.

\begin{tabular}{lccc}
\hline & Female $(\mathrm{n}=40)$ & Males $(\mathrm{n}=18)$ & Overall $(\mathrm{n}=58)$ \\
\hline Age $(\mathrm{yrs})$ & $69.7 \pm 5.3$ & $70.0 \pm 5.9$ & $69.8 \pm 4.9$ \\
Height $(\mathrm{cm})$ & $161.9 \pm 5.0$ & $175.9 \pm 6.7$ & $166.3 \pm 8.6$ \\
Mass $(\mathrm{kg})$ & $69.7 \pm 5.3$ & $88.1 \pm 21.0$ & $76.4 \pm 18.4$ \\
BMI $\left(\mathrm{kg} \cdot \mathrm{m}^{-2}\right)$ & $26.9 \pm 4.8$ & $28.3 \pm 5.2$ & $27.3 \pm 4.9$ \\
\hline
\end{tabular}

3

4 
Table 2 (on next page)

Frequency statistics for each genotype for all participants $(n=58)$. 
2 Table 2. Frequency statistics for each genotype for all participants $(\mathbf{n}=\mathbf{5 8})$.

\begin{tabular}{lccc}
\hline \multicolumn{1}{c}{ Polymorphism } & Genotype & Frequency & $\begin{array}{c}\text { Hardy Weinberg Equilibrium } \\
\text { (p-value) }\end{array}$ \\
\hline ACE intron 16 & DD & 0.295 & 1.00 \\
insertion/deletion & ID & 0.492 & \\
& II & 0.213 & 0.990 \\
UCP2 G-866A & GG & 0.344 & \\
& GA & 0.541 & \\
& AA & 0.115 & \\
& & &
\end{tabular}

3

4 


\section{Table 3 (on next page)}

Baseline (pre-training) physical abilities according to ACE, adjusted for age and gender. In the final two columns, the upper row for each assessment provides the $p$ value and in the lower row the value in parentheses is the effect size. Higher scores are indicative of better performance in all tests with the exception of the $8 \mathrm{ft} U p$ and Go in which a smaller score indicates better performance. * Indicates a significant difference between genotype groups for each specific assessment. \#Referent group 
2 Table 3. Baseline (pre-training) physical abilities according to ACE, adjusted for age and gender.

3

\begin{tabular}{|c|c|c|c|c|c|c|c|c|c|c|}
\hline \multirow[t]{2}{*}{ Assessment } & \multicolumn{2}{|c|}{ ACE II\# } & \multicolumn{2}{|c|}{ ACE ID } & \multicolumn{2}{|c|}{ ACE DD } & \multicolumn{2}{|c|}{$\mathrm{ACE}$ ID/DD } & \multirow{2}{*}{$\begin{array}{c}\text { IIvIDvDD } \\
\text { p-value } \\
\text { (effect size) }\end{array}$} & \multirow{2}{*}{$\begin{array}{c}\text { IIvID/DD } \\
\text { p-value } \\
\text { (effect size) }\end{array}$} \\
\hline & $\mathrm{n}$ & $\mathrm{M} \pm \mathrm{SD}$ & $\mathrm{n}$ & $\mathrm{M} \pm \mathrm{SD}$ & $\mathrm{n}$ & $\mathrm{M} \pm \mathrm{SD}$ & $\mathrm{n}$ & $\mathrm{M} \pm \mathrm{SD}$ & & \\
\hline 6 min walk (m) & 7 & $458 \pm 28.7^{*}$ & 26 & $531 \pm 14.6$ & 13 & $576 \pm 20.7$ & 39 & $546 \pm 12.1 *$ & $\begin{array}{c}0.339 \\
(0.659)\end{array}$ & $\begin{array}{c}0.008 \\
(0.614)\end{array}$ \\
\hline 8ft Up \& Go (s) & 7 & $6.45 \pm 0.48^{*}$ & 26 & $4.42 \pm 0.24$ & 13 & $4.39 \pm 0.34$ & 39 & $4.41 \pm 0.19^{*}$ & $\begin{array}{c}0.219 \\
(0.779)\end{array}$ & $\begin{array}{l}<0.001 \\
(0.781)\end{array}$ \\
\hline $\begin{array}{l}\text { Sit to Stand } \\
\text { (repetitions) }\end{array}$ & 7 & $12.4 \pm 2.23$ & 26 & $13.8 \pm 1.14$ & 13 & $17.9 \pm 1.61$ & 39 & $15.2 \pm 0.95$ & $\begin{array}{c}0.269 \\
(0.726)\end{array}$ & $\begin{array}{c}0.268 \\
(0.565)\end{array}$ \\
\hline $\begin{array}{l}\text { Bicep curls } \\
\text { (repetitions) }\end{array}$ & 7 & $15.4 \pm 1.41$ & 26 & $16.2 \pm 0.72$ & 13 & $17.8 \pm 1.01$ & 39 & $16.7 \pm 0.61$ & $\begin{array}{c}0.726 \\
(0.273)\end{array}$ & $\begin{array}{c}0.421 \\
(0.684)\end{array}$ \\
\hline $\begin{array}{l}\text { Handgrip } \\
\text { strength }(\mathrm{kg})\end{array}$ & 8 & $30.9 \pm 3.01$ & 29 & $31.7 \pm 1.48^{*}$ & 14 & $29.3 \pm 2.18^{*}$ & 43 & $30.9 \pm 1.21$ & $\begin{array}{l}<0.001 \\
(0.786)\end{array}$ & $\begin{array}{c}0.994 \\
(0.001)\end{array}$ \\
\hline $\begin{array}{l}\text { Purdue } \\
\text { Pegboard (pegs) }\end{array}$ & 8 & $11.4 \pm 2.01$ & 29 & $14.9 \pm 0.99$ & 13 & $11.7 \pm 1.62$ & 42 & $14.0 \pm 0.85$ & $\begin{array}{c}0.451 \\
(0.547)\end{array}$ & $\begin{array}{c}0.239 \\
(0.375)\end{array}$ \\
\hline
\end{tabular}

4 In the final two columns, the upper row for each assessment provides the p value and in the lower row the value in parentheses is the effect

5 size. Higher scores are indicative of better performance in all tests with the exception of the 8ft Up and Go in which a smaller score indicates

6 better performance. * Indicates a significant difference between genotype groups for each specific assessment.

$7 \quad$ \#Referent group 


\section{Table 4(on next page)}

Baseline (pre-training) physical abilities stratified by UCP2 G-886A polymporhism, adjusted for age and gender

In the final column, the upper row for each assessment provides the $p$ value and in the lower row the value in parentheses is the effect size. Higher scores are indicative of better performance in all tests with the exception of the $8 \mathrm{ft} \mathrm{Up}$ and $\mathrm{Go}$ in which a smaller score indicates better performance. * Indicates a significant difference between genotype groups for each specific assessment. \#Referent group 
2 Table 4. Baseline (pre-training) physical abilities stratified by UCP2 G-886A polymporhism, adjusted 3 for age and gender.

4

$$
5
$$

6

7

8

9

10

11

12

13

14

15

16

17

18

19

20

21

22

23

24

25

26

27

28 heses is the effect size. Higher scores are indicative of better performance in all tests with the exception

29 of the $8 \mathrm{ft} \mathrm{Up}$ and Go in which a smaller score indicates better performance. * Indicates a significant

30 difference between genotype groups for each specific assessment.

31 \#Referent group 


\section{Table 5 (on next page)}

Changes in physical measures for each ACE allele after 12 weeks physical training intervention on older adults

In the final two columns, the upper row for each assessment provides the $p$ value and in the lower row the value in parentheses is the effect size. Positive scores are indicative of improvements in performance in all tests with the exception of the $8 \mathrm{ft}$ Up and Go in which a negative change indicates better performance. \#Referent group 
2 Table 5. Changes in physical measures for each ACE allele after 12 weeks physical training intervention on older adults.

6 min walk (m) $\quad 6 \quad 28.8 \pm 14.5 \quad 26 \quad 44.2 \pm 7.23 \quad 13 \quad 44.5 \pm 10.0 \quad 39 \quad 44.3 \pm 5.95 \quad 0.607(0.533) \quad 0.315(0.703)$

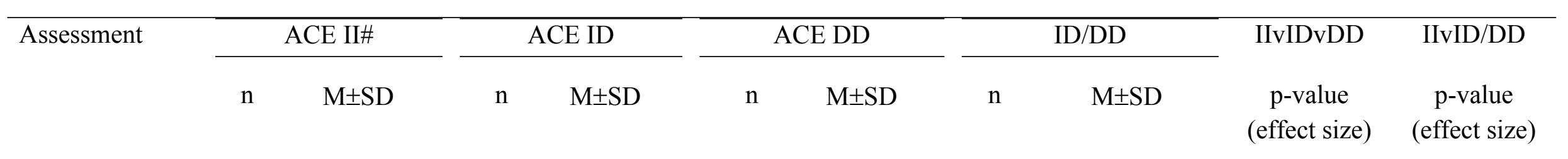

\begin{tabular}{lcccccccccc}
\hline 8ft Up \& Go (s) & 6 & $-0.55 \pm 0.30$ & 26 & $-0.23 \pm 0.15$ & 13 & $-0.32 \pm 0.20$ & 39 & $-0.26 \pm 0.12$ & $0.621(0.878)$ & $0.365(0.916)$ \\
$\begin{array}{l}\text { Sit to Stand } \\
\text { (repetitions) }\end{array}$ & 6 & $1.62 \pm 1.58$ & 26 & $3.43 \pm 0.79$ & 13 & $1.25 \pm 1.09$ & 39 & $2.71 \pm 0.67$ & $0.217(0.809)$ & $0.536(0.525)$ \\
$\begin{array}{l}\text { Bicep curls } \\
\text { (repetitions) }\end{array}$ & 6 & $-0.02 \pm 1.14$ & 26 & $1.77 \pm 0.57$ & 13 & $2.50 \pm 0.79$ & 39 & $2.01 \pm 0.47$ & $0.198(0.416)$ & $0.099(0.923)$ \\
$\begin{array}{l}\text { Handgrip } \\
\text { strength (kg) }\end{array}$ & 7 & $1.64 \pm 1.56$ & 29 & $0.78 \pm 0.79$ & 13 & $2.50 \pm 1.15$ & 42 & $1.31 \pm 0.68$ & $0.450(0.043)$ & $0.853(0.054)$ \\
$\begin{array}{l}\text { Purdue } \\
\text { Pegboard (pegs) }\end{array}$ & 7 & $-0.31 \pm 0.83$ & 29 & $0.62 \pm 0.42$ & 13 & $1.41 \pm 0.63$ & 42 & $0.85 \pm 0.37$ & $0.234(0.965)$ & $0.189(0.769)$
\end{tabular}

3 In the final two columns, the upper row for each assessment provides the $\mathrm{p}$ value and in the lower row the value in parentheses is the effect 4 size. Positive scores are indicative of improvements in performance in all tests with the exception of the $8 \mathrm{ft}$ Up and Go in which a negative 5 change indicates better performance.

6 \#Referent group 
Table 6(on next page)

Changes in physical measures for each UCP2 G-886A allele after 12 weeks physical training intervention in older adults.

In the final column, the upper row for each assessment provides the $\mathrm{p}$ value and in the lower row the value in parentheses is the effect size. Positive scores are indicative of improvements in performance in all tests with the exception of the $8 \mathrm{ft}$ Up and Go in which a negative change indicates better performance. \#Referent group 
Table 6. Changes in physical measures for each UCP2 G-886A allele after 12 weeks physical training intervention in older adults.

3

\begin{tabular}{|c|c|c|c|c|c|c|c|}
\hline \multirow[t]{2}{*}{ Assessment } & \multicolumn{2}{|c|}{ UCP2 G-886A AA } & \multicolumn{2}{|c|}{ UCP2 G-886A GA } & \multicolumn{2}{|c|}{ UCP2 G-886A GG\# } & \multirow{2}{*}{$\begin{array}{c}\text { AAvGAvGG } \\
\text { p-value (effect size) }\end{array}$} \\
\hline & $\mathrm{n}$ & $\mathrm{M} \pm \mathrm{SD}$ & $\mathrm{n}$ & $\mathrm{M} \pm \mathrm{SD}$ & $\mathrm{n}$ & $\mathrm{M} \pm \mathrm{SD}$ & \\
\hline 6 min walk (m) & 5 & $39.1 \pm 16.9$ & 23 & $41.4 \pm 7.52$ & 17 & $44.3 \pm 9.15$ & $0.949(0.397)$ \\
\hline 8ft Up \& Go (s) & 5 & $0.05 \pm 0.31^{*}$ & 23 & $-0.10 \pm 0.14$ & 17 & $-0.68 \pm 0.17^{*}$ & $0.023(0.714)$ \\
\hline Sit to Stand (repetitions) & 5 & $2.93 \pm 1.89$ & 23 & $2.85 \pm 0.84$ & 17 & $2.01 \pm 1.02$ & $0.789(0.447)$ \\
\hline Bicep curls (repetitions) & 5 & $1.00 \pm 1.36$ & 23 & $1.95 \pm 0.61$ & 17 & $1.60 \pm 0.74$ & $0.799(0.200)$ \\
\hline Handgrip strength (kg) & 5 & $0.72 \pm 1.94$ & 26 & $1.07 \pm 0.82$ & 18 & $2.02 \pm 1.03$ & $0.708(0.447)$ \\
\hline Purdue Pegboard (pegs) & 5 & $1.50 \pm 1.05$ & 26 & $0.72 \pm 0.45$ & 18 & $0.38 \pm 0.56$ & $0.622(0.319)$ \\
\hline
\end{tabular}

4

In the final column, the upper row for each assessment provides the $p$ value and in the lower row the value in parentheses is the effect size.

Positive scores are indicative of improvements in performance in all tests with the exception of the 8ft Up and Go in which a negative change indicates better performance.

\#Referent group 